\title{
La mise en valeur du milieu local en moyenne montagne : l'exemple des réseaux secondaires du Haut-Beaujolais (1881-1969)
}

Frédéric Toublanc

\section{CpenEdition}

\section{Journals}

Édition électronique

URL : https://journals.openedition.org/rhcf/2070

DOI : 10.4000/rhcf.2070

Éditeur

Rails \& histoire

Édition imprimée

Date de publication : 2 mai 2002

Pagination : 297-306

ISBN : 00996-9403

ISSN : 0996-9403

Référence électronique

Frédéric Toublanc, «La mise en valeur du milieu local en moyenne montagne : l'exemple des réseaux secondaires du Haut-Beaujolais (1881-1969) », Revue d'histoire des chemins de fer [En ligne], 24-25 | 2002, mis en ligne le 20 avril 2015, consulté le 22 avril 2022. URL : http://journals.openedition.org/ rhcf/2070 ; DOl : https://doi.org/10.4000/rhcf.2070 


\section{La mise en valeur du milieu local en moyenne montagne : I'exemple des réseaux secondaires du Haut-Beaujolais (1881-1969)}

L'étude porte sur trois lignes secondaires embranchées sur la ligne d'intérêt général Roanne à Lyon entre 1881 et 1907 ; on s'autorisera néanmoins à d'utiles comparaisons avec la ligne voisine des Chemins de fer du Beaujolais (1901) et son prolongement naturel avec le réseau interdépartemental du Rhône-Saône-et-Loire (1911).

\section{Un microcosme géographique florissant}

Le Haut-Beaujolais forme un môle montagneux dans la partie orientale du Massif central, séparant les bassins de la Loire et de la Saône. De hautes surfaces granitiques comprises entre 600 et $900 \mathrm{~m}$ dessinent des lanières entaillées par trois vallées d'orientation méridienne, s'étalant d'Ouest en Est : la Trambouze, le Reins ${ }^{1}$ et l'Azergues. C'est un milieu difficile, marqué par un climat sévère, des sols médiocres, de vastes forêts de hêtres et sapins (fig. 1). Néanmoins l'ensemble connait une forte humanisation (des densités de plus de 150 habitants au $\mathrm{km}^{2}$ ) du fait d'une tradition manufacturière vieille de près de trois siècles (toiles de lin et de chanvre, cotonnes et soieries) dynamisée sous le Second Empire et surtout après la guerre de 1870 qui, en accroissant la demande du fait de la disparition de la concurrence alsacienne, accélère la mécanisation (développement de l'originale industrie de la couverture à Cours, centre lainier d'importance nationale). Il en résulte une forte concentration urbaine au long des vallées - vraies rues d'usines aux toits en sheds et hautes cheminées - et l'essor spectaculaire de pôles urbains comme Thizy, Cours, Amplepuis ou Tarare, contrôlés par de véritables dynasties d'actifs et ingénieux capitaines d'industrie.

De tels progrès génèrent un formidable développement de la circulation des marchandises - charbonnages, matières premières textiles, matériaux de construction - pondéreux que le réseau routier hérité de l'Ancien Régime n'est pas à même d'absorber, tant on reste tributaire de chemins que les municipalités dénoncent comme « exécrables, car trop étroits et constamment défoncés par des voitures trop lourdes et une circulation exagérée ». Cet enclavement n'est que peu modifié par l'arrivée du chemin de fer construit par la Cie P.L.M. avec la transversale 
Revue d'histoire des chemins de fer 24-25 (printemps - automne 2001)

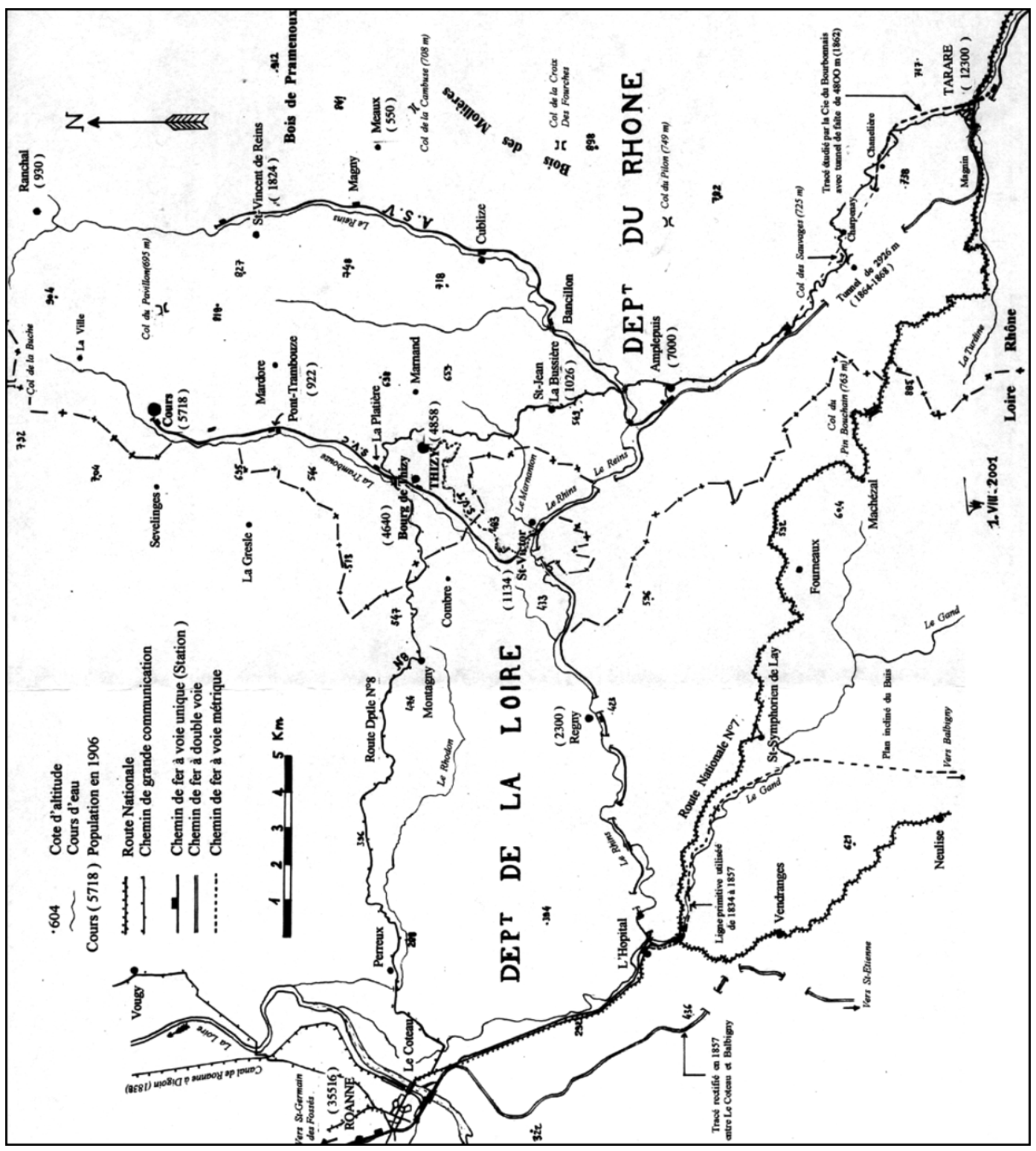

Figure 1 : Le Haut-Beaujolais occidental : carte de situation en 1907. 
Roanne-Lyon par Tarare achevée en 1868. Grande est alors la déception des populations et des industriels face à cette ligne comportant un tracé accidenté, des profils sévères $(26 \mathrm{~mm} / \mathrm{m})$, un long tunnel de faite $(3$ $\mathrm{km}$ ), une voie unique jusqu'à Amplepuis, une situation enfin trop excentrée au Sud, obligeant à de longs et coûteux transbordements en gare de StVictor-Thizy (la ligne Paray-Le Monial à Lozanne, ouverte en 1900 par le Val d'Azergues, ne répondra pas davantage aux attentes, car évitant la région par le Nord-Est). Aussi est-ce afin de combler les vides laissés par le grand réseau qu'émergent très vite des projets visant à greffer sur l'axe P.L.M. une antenne ferroviaire "d'intérêt local et industriel », présentée comme «une question de vie ou de mort pour l'industrie locale».

\section{Des greffes ferroviaires souvent précoces et exemplaires}

On notera l'étroite concomitance entre le dépôt des premières études (1866) et le vote récent de la loi Migneret de juillet 1865 qui libère les énergies locales en favorisant la mise en place d'un second réseau laissé à l'initiative des collectivités. Si l'on mentionne pour mémoire les rivalités de clocher entre Cours et Thizy, le manque de réalisme de certains avant-projets (tramway à traction animale, chemin de fer routier à crémaillère, ligne susceptible d'être prolongée pour se raccorder au Nord à Chaufailles avec la future ligne de Paray à Moulins), les atermoiements des pouvoirs publics (méfiance du département du Rhône échaudé par la déconfiture rapide d'une «mignerette » voisine, la courte ligne du Beaujeu à Belleville ouverte en 1869 et reprise par le P.L.M. en 1878) et l'incontournable hostilité du P.L.M., on comprendra qu'il faille attendre 1876 et 1877 pour qu'on arrête l'octroi de...deux concessions embranchées à St-Victor P.L.M. La première, à voie normale, remonte la vallée de la Trambouze pour atteindre Cours, la seconde, à voie métrique, se limite à la desserte de Thizy. La vallée du Reins devra attendre l'accord de $1898 \ldots$ année où le département du Rhône entame enfin les premiers travaux des Chemins de fer du Beaujolais, après vingt années de tergiversations relatives au choix des lignes (liaison Villefranche à Tarare et Villefranche à Monsols par Beaujeu, en attendant un prolongement ultérieur vers La Clayette P.L.M. ).

Le tableau 1 permet de saisir le cadre juridique de chaque compagnie : régime de la loi Migneret pour le Saint-Victor-Cours ou S.V.C. (d'où une durée de concession de 99 ans et une grande liberté de manœuvre pour le choix du matériel d'exploitation), régime de la loi de juin 1880 pour le Saint-Victor-Thizy (S.V.T.) et l'Amplepuis-Saint-Vincentde-Reins (A.S.V.) ; financement par des capitaux privés (constitution de sociétés anonymes) et publics (subventions versées par les départements et l'État $)^{2}$. 
Le choix de l'écartement s'avére décisif pour l'avenir de ces lignes. Tandis que la voie large s'est imposée dès le départ pour les industriels de Cours, évitant du même coup la rupture de charge avec le grand réseau, c'est à la voie étroite plus adaptée à la desserte de type tramway que s'en remet le concessionnaire du S.V.T. Et c'est fort de la réussite du S.V.C. que le Rhône opte en 1898 pour la voie large sur l'A.S.V. et ce, contre l'avis du Conseil d'État, ce qui n'empêchera pas le concessionnaire de réaliser une infrastructure légère.

On relèvera pourtant que le dépassement systématique du maximum d'établissement de tous ces réseaux pose le problème de la trop fréquente sous-évaluation des coûts tant par les concessionnaires que par les autorités de tutelle. On arrive ainsi à des prix de revient au $\mathrm{km}$ de $112000 \mathrm{~F}$ pour le S.V.T., $116000 \mathrm{~F}$ pour l'A.S.V. et plus de $180000 \mathrm{~F}$ pour le S.V.C.!

\section{Des moyens mis en œuvre adaptés aux ambitions}

Les hommes. Il faut d'abord souligner le rôle déterminant des concepteurs, soit: pour le S.V.C., l'industriel de Bourg-de-Thizy, Poizat-Coquard, et son bras droit l'ingénieur des Ponts et Chaussées Millat, intermédiaire efficace avec les ateliers lyonnais de La Buire; pour le S.V.T., l'ingénieur civil lyonnais Ailloud. Dans les deux cas, on souligne dès l'époque la "réussite technique » des deux entreprises, en total contraste avec les résultats désastreux obtenus par les peu scrupuleux entrepreneurs de l'A.S.V., Vouillon et son gendre Vernet.

La direction de l'exploitation est confiée à des hommes compétents souvent recrutés parmi des retraités du P.L.M. (cas des S.V.C. et S.V.T.), mais aussi parmi des personnels provenant de réseaux secondaires voisins (Tramways de l'Ain, Rhône-Saône-et-Loire, Chemins de fer du Beaujolais). Et c'est forts de leur expérience qu'ils rédigent des règlements généraux très sophistiqués, car souvent calqués sur ceux des grands réseaux.

Les personnels, recrutés et formés sur place pour l'essentiel, atteignent parfois des effectifs pléthoriques à l'image du P.L.M. : 3,57 agents par km exploité au S.V.T., jusqu'à cinq au S.V.C. (contre 1,36 à l'A.S.V. et aux C.F.B.) (tabl. 2). Il en résulte souvent un enracinement sur plusieurs générations, point très bénéfique pour la bonne marche du réseau, mais obérant les comptes d'exploitation dans les années d'après-guerre. Un dernier trait est celui de l'étroite solidarité entre les agents des réseaux, en particulier au niveau syndical : les récriminations sont ainsi vives en ce qui concerne les revendications salariales, les personnels de l'A.S.V. et du S.V.T. voulant être alignés sur la grille des traitements du S.V.C. ${ }^{3}$. 


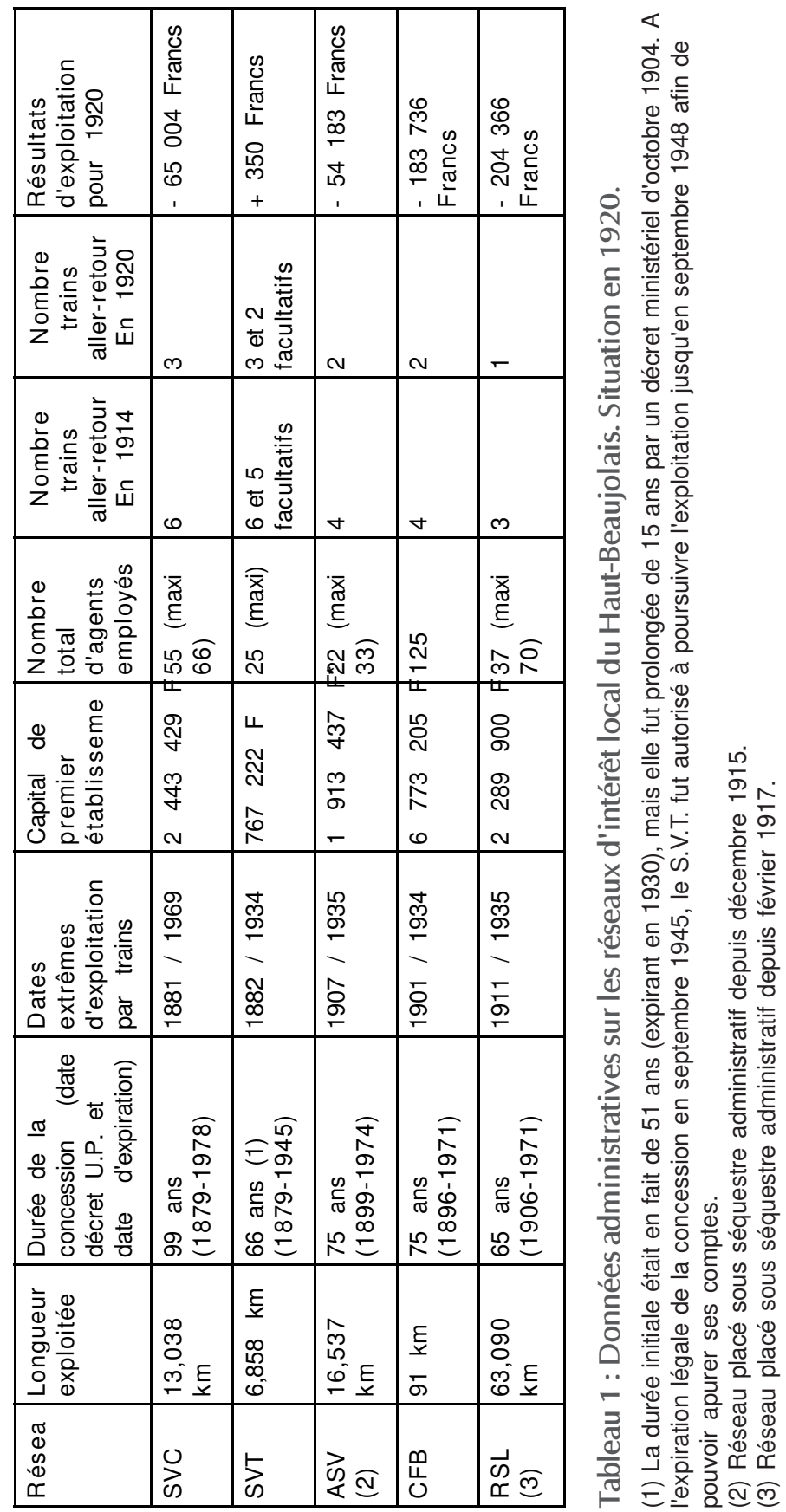




\begin{tabular}{|c|c|c|c|c|c|c|}
\hline $\begin{array}{l}\frac{1}{2} \\
\overline{0} \\
\overline{0} \\
\frac{0}{0}\end{array}$ & $\begin{array}{l}\infty \\
\check{0} \\
0 \\
\mathbb{\pi} \\
3\end{array}$ & $\sigma$ & $\stackrel{10}{m}$ & $\stackrel{20}{\longrightarrow}$ & $\stackrel{\circ}{\forall}$ & $\stackrel{\infty}{\infty}$ \\
\hline 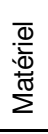 & 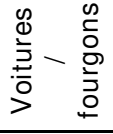 & $\begin{array}{l}+ \\
- \\
\end{array}$ & $\begin{array}{c}1 \\
- \\
\infty\end{array}$ & $\begin{array}{l}\sim \\
+ \\
+\end{array}$ & $\begin{array}{l}0 \\
\stackrel{\rho}{\circ}\end{array}$ & $\begin{array}{l}0 \\
- \\
\infty\end{array}$ \\
\hline \multirow{6}{*}{ 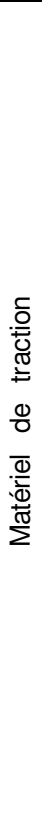 } & 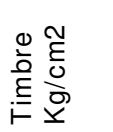 & $\sigma$ & $\begin{array}{l}\stackrel{0}{0} \\
\stackrel{5}{\sim}\end{array}$ & $\begin{array}{l}\cong \\
\simeq \\
+ \\
0 \\
\infty\end{array}$ & $\stackrel{\sim}{\sim}$ & $\stackrel{m}{-}$ \\
\hline & 응 $\frac{0}{0} \frac{0}{\frac{0}{\pi}}$ & $\stackrel{+}{N}$ & $\stackrel{\vec{\sigma}}{\sigma}$ & ్ָల & $\stackrel{+}{\bar{N}}$ & 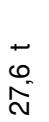 \\
\hline & $\begin{array}{l}0 \\
0 \\
0 \\
0 \\
0.0 \\
0.0\end{array}$ & $\frac{+}{\bar{N}}$ & $\begin{array}{l}\stackrel{-}{N} \\
\stackrel{n}{n}\end{array}$ & $\stackrel{\stackrel{\oplus}{N}}{\stackrel{N}{N}}$ & $\stackrel{+}{\infty}$ & $\begin{array}{l}\dot{+} \\
\dot{N}\end{array}$ \\
\hline & $\begin{array}{l}\bar{y} \\
0 \\
0 \\
0 \\
\pm \\
0 \\
0 \\
0 \\
0\end{array}$ & $\begin{array}{l}\frac{1}{0} \\
\frac{0}{0} \\
\frac{1}{0} \\
\frac{1}{0} \\
\infty\end{array}$ & 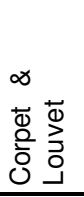 & 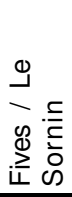 & $\begin{array}{l}\frac{7}{0} \\
\frac{1}{0} \\
\stackrel{-}{0} \\
0\end{array}$ & $\begin{array}{l}\frac{1}{0} \\
\stackrel{3}{0} \\
.0\end{array}$ \\
\hline & $\frac{0}{2}$ & $\nabla$ & $m$ & $m$ & $\stackrel{*}{\sigma}$ & $\stackrel{*}{*}$ \\
\hline & $\begin{array}{l}\stackrel{0}{0} \\
\stackrel{0}{\Sigma} \\
⺊\end{array}$ & '. & ๖్ & ๖్ & ڤ్ & 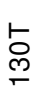 \\
\hline \multirow{3}{*}{$\frac{0}{0}$} & 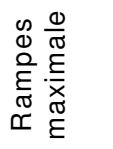 & $\begin{array}{l}\frac{\xi}{\xi} \\
\text { है } \\
\stackrel{N}{m}\end{array}$ & $\begin{array}{l}\frac{\xi}{\xi} \\
\xi \\
\stackrel{\rho}{\infty}\end{array}$ & $\begin{array}{l}\stackrel{\xi}{E} \\
\text { E } \\
\stackrel{\infty}{N}\end{array}$ & $\frac{\xi}{\xi}$ & $\begin{array}{l}\sum_{\xi}^{\xi} \\
\xi \\
\stackrel{\rho}{\rho}\end{array}$ \\
\hline & 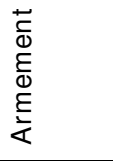 & 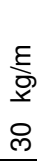 & 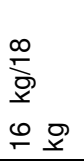 & $\begin{array}{l}\frac{\varepsilon}{D} \\
\stackrel{2}{ } \\
\stackrel{N}{N}\end{array}$ & $\xi$ & $\begin{array}{l}\frac{\varepsilon}{D} \\
\stackrel{N}{o} \\
\stackrel{N}{ }\end{array}$ \\
\hline & 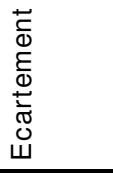 & $\begin{array}{l}\varepsilon \\
\stackrel{L}{\tilde{J}} \\
\stackrel{+}{-}\end{array}$ & $\begin{array}{l}\varepsilon \\
\tau\end{array}$ & 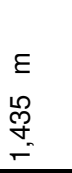 & $\varepsilon$ & $\begin{array}{l}\varepsilon \\
-\end{array}$ \\
\hline $\begin{array}{l}\widetilde{0} \\
0 \\
\infty \\
\infty \\
\simeq\end{array}$ & & ळ & $\stackrel{5}{5}$ & 娄 & 虫 & $\stackrel{\vec{\omega}}{\widetilde{\simeq}}$ \\
\hline
\end{tabular}


Les infrastructures (tabl. 2). Le tracé est celui d'une ligne d'intérêt général pour le S.V.C. (courbes jamais inférieures à $300 \mathrm{~m}$ ), celui d'un tramway en accotement de route pour le S.V.T. (courbes serrées et souvent très rapprochées); il est en revanche réalisé à l'économie pour l'A.S.V., c'est à dire avec des critères techniques relevant plus de la voie étroite que de la voie normale. Les bâtiments sont souvent, quant à eux, d'inspiration libre, ce qui n'exclut pas le recours au monumental (gares de Cours et de Thizy).

Le matériel roulant. Les choix de départ ont souvent été dictés en jouant sur le réseau de relations établi avec des compagnies privées voisines (en particulier la Cie des Dombes et du Sud-Est pour les dirigeants du S.V.C.) et les firmes locales de construction de matériel ferroviaire (La Buire et Pinguely à Lyon, Schneider au Creusot, Les Ateliers du Sornin à La Clayette), ce qui n’a pas empêché le recours à des constructeurs plus lointains, mais confirmés (Cail et Corpet pour le S.V.T.), voire l'acquisition de matériel d'occasion auprès des grands réseaux (Cie du Nord pour l'A.S.V.). Globalement, le matériel de traction adopté s'est révélé fiable, s'en remettant au classique type $030 \mathrm{~T}$ acquis neuf de construction, sauf pour l'A.S.V. qui se dote d'un matériel de près de 40 ans d'âge, donc déjà obsolète et vite fatiguét. L'attachement à la vapeur est resté de règle durant toute l'existence de nos trois réseaux, alors que C.F.B. et R.S.L ont eu recours sur le tard - sans grand succès du reste - à des prototypes « d'auto-rails ».

Les voitures de voyageurs sont de «type américain », c'est-à-dire avec accès par des plate-formes d'extrémité, soit d'occasion exDombes-P.L.M. pour les réseaux S.V.C. et A.S.V., soit neuves pour les réseaux à voie métrique, de marque Buire pour le S.V.T., Blanc-Misseron pour le C.F.B. ou Le Sornin pour le R.S.L. ${ }^{5}$. Choix du neuf en revanche pour les wagons de marchandises, car c'est de ce transport que ces réseaux à vocation d'abord industrielle attendent l'essentiel de leurs recettes.

Ce matériel est entretenu dans des ateliers de taille modeste, mais bien dirigés et aptes à faire face à toutes les situations. Mentionnons ainsi le cas des machines $130 \mathrm{~T}$ du R.S.L., entretenues avec zèle par le chef mécanicien Berthelon de 1913 à 1928, lequel prendra en charge les rutilantes $030 \mathrm{~T}$ du S.V.C. de 1930 à 1949, engins dont la longévité atteindra les 80 ans! Dans le cas contraire, les prestations médiocres des engins sont plus à mettre au compte des insuffisances financières des compagnies que de l'incompétence ou de la négligence des hommes ('A.S.V. doit même recourir à la location de locomotives «étrangères » pour pallier la mise hors service de deux de ses machines). 


\section{Une exploitation qui participe pleinement à la prospérité de la région}

Organisation générale. On utilise le classique système des rames MV (marchandises-voyageurs), avec quatre à six aller-retour journaliers et des trains supplémentaires les jours de marchés ou foires. Mention particulière pour le S.V.T. qui comporte jusqu'à 11 aller-retour en navettes de type tramway (avec la machine retenant la rame à la descente), et surtout pour le S.V.C. qui met en marche de fréquents trains spéciaux de marchandises, avec utilisation de la double traction en pousse non attelée (autorisant des charges de 160 à $180 \mathrm{t}$ sur des rampes de $23 \mathrm{~mm} / \mathrm{m}$ ). Les besoins deviennent même tels qu'on met en service un « train croiseur » entre 1913 et 1938, système assez rare sur une ligne à voie unique de... $13 \mathrm{~km}$ de longueur. Pour compléter ces traits originaux, ajoutons que les passages à niveau y sont gardés - alors que la ligne est classée d'intérêt local - et que la desserte voyageurs comporte d'origine les trois classes.

Les résultats. Le trafic marchandises est l'exact reflet de l'activité industrielle de la région : combustibles et matières premières à la montée, produits finis à la descente. Si l'A.S.V. tourne autour des $10000 \mathrm{t}$ annuelles, le S.V.T. atteint les 30000 t durant les années d'avant 1914, tandis que le S.V.C. se maintient le plus souvent entre 60 et $70000 \mathrm{t}$ (avec un pic à 83700 t en 1926). Pour les voyageurs, l'A.S.V. transporte une moyenne de 40000 personnes par an, le S.V.T. 60000 et le S.V.C de 130 à 150 000. On relèvera le gonflement artificiel de ces chiffres durant les périodes de guerre : jusqu'à 58000 voyageurs en 1919 pour l'A.S.V. et... 163988 pour le S.V.C. (lequel remontera à 105000 en 1943).

Les bilans sont donc très largement - et durablement excédentaires pour le S.V.C. : les dividendes sont versés aux actionnaires durant 44 exercices durant la période faste comprise entre 1887 et 1942, avec remboursement échelonné du capital à partir de 1911, selon les volontés du conseil d'administration de cette société de famille. Résultats eux aussi excédentaires pour le S.V.T. durant la période 1889-1929, ce qui lui permet de surmonter les difficultés des premières années, allant jusqu'à reconstruire trois fois les installations de la gare de Thizy. C'est la concurrence routière des années 1925 et surtout la crise économique à partir de 1932 qui creusent le déficit. Seul le S.V.C. parvient à s'en sortir en comprimant les charges d'exploitation (relèvement des tarifs, suppression de trains, réduction des effectifs au gré des départs en retraites) et en observant une gestion prudente. Situation très différente pour l'A.S.V., puisque vrai fiasco financier du début à la fin, victime tant 
des affairistes qui l'ont conçu trop tardivement que d'une conjoncture peu favorable. Mentionnons ainsi le choc de la guerre qui entraîne la mobilisation des personnels et la fermeture de la ligne dès le 24 août 1914 (on la remet en service à la demande des autorités militaires le $1^{\text {er }}$ août 1916), l'usure rapide du matériel roulant et le délabrement de la voie dont les traverses ne sont pas traitées. La gestion se fait sous séquestre administratif dès 1915 et le rachat par le département intervient en 1927. Néanmoins, à l'examen des 10000 t encore transportées au début des années 1930 - essentiellement du bois - on peut penser que le département du Rhône aurait pu maintenir cette ligne à voie normale pour le trafic marchandises, même si l'état précaire du matériel avait de quoi le décourager.

Le S.V.T. abandonne l'exploitation par trains dès 1934 suite au transfert sur route de son activité, ce qui va lui permettre de perdurer jusqu'à la date légale d'expiration de sa concession prolongée de trois ans, soit en 1948.

Demeuré l'unique survivant de nos secondaires du HautBeaujolais, le S.V.C. réussit à surmonter l'épreuve de la Deuxième Guerre mondiale, délivrant encore plus de 30000 billets de voyageurs jusqu'en 1950, moins de 15000 à partir de 1956. C'est donc le 31 juillet 1960 qu'est abandonné le service voyageurs - comprenant toujours les trois classes - et que l'on gare les dernières «cages à poules » remontant pour certaines au Second Empire. Les trois dernières locomotives sont éteintes l'année suivante, ce qui n'empêche pas notre S.V.C. de réussir le tour de force sans doute unique en France de continuer la desserte marchandises à partir d'avril 1961 sans aucun matériel propre puisqu'il loue les services d'un diesel BB 63000 de la SNCF... On a donc un matériel SNCF roulant sur une ligne privée, pour le compte d'une compagnie privée, à l'inverse de ce qui se passe à l'époque sur la ligne SNCF Avallon-Clamecy affermée aux C.F.T.A. pour l'exploiter. Mais cette survie reste artificielle et l'érosion du trafic ne peut être stoppée, aggravée par deux facteurs: d'abord la volonté de la SNCF de se débarrasser de ce "gêneur» en lui retirant en 1966 le trafic détail et petits colis, soit $75 \%$ de son trafic (le fameux régime de « desserte en surface » instauré dès 1962 par la coordination rail-route), ensuite la crise textile de 1965 qui frappe de plein fouet des maisons souvent centenaires aux structures familiales vieillies (le tissage cotonnier de Thizy perd ainsi les 2/3 de ses effectifs). C'est le 31 janvier 1969 que le S.V.C. jette l'éponge, non sans avoir remboursé le reliquat du capital aux derniers actionnaires! 
On retiendra de l'histoire de nos lignes du Haut-Beaujolais particulièrement le S.V.C. et le S.V.T. - que si leurs faibles performances horaires et leur matériel vieillissant les firent à la longue assimiler à des tacots, ils n'en restent pas moins des exemples de secondaires qui, parce qu'ils avaient vu le jour dès l'aube de la Troisième République pour répondre à des besoins économiques réels et non dans un dessein électoral - comme maintes lignes ouvertes au tournant du XXe siècle surent pleinement faire la démonstration de leur viabilité, dignes compléments des grands réseaux dont ils constituaient les affluents naturels. Un siècle plus tard, leur empreinte demeure du reste vivace dans la mémoire locale comme dans les paysages, et le patrimoine national peut s'enorgueillir de compter dans son héritage deux locomotives Schneider du S.V.C. et une Cail du S.V.T., sans doute la plus vieille machine de ce type conservée en France (1880).

\section{Notes}

Voir, pour une histoire complète de ces lignes, l'ouvrage: Les Trains de la Trambouze : les chemins de fer de Saint-Victor à Cours et de Saint-Victor à Thizyy, Presses et Éditions ferroviaires, 2000, $224 \mathrm{p}$.

1- Rivière dont le nom s'orthographie Reins dans le Rhône pour devenir Rhins dans la Loire.

2- Subvention de 1 million pour le S.V.C. (dont $300000 \mathrm{~F}$ au titre du concours des industriels), 1 million pour les C.F.B., 1,5 millions pour l'A.S.V., mais aucune pour le S.V.T., hormis une « contribution gracieuse » de $40000 \mathrm{~F}$ allouée en 1883 afin de combler des dépenses imprévues sans avoir à toucher au fond de réserve de la compagnie.

3- Les personnels du S.V.C. ont de leur côté toujours pris pour référence les hauts salaires versés au réseau de l'Est de Lyon (538 agents en 1925 pour $93 \mathrm{~km}$ de lignes)

4- Mentionnons pour mémoire que le réseau C.F.B. a toujours souffert du manque d'adhérence de ses trop légères $030 \mathrm{~T}$ Pinguely, alors qu'il aurait pu les transformer en type 130, opération réalisée avec profit par les C.F.C. de la Loire en 1909.

5- Rappelons l'inénarrable bévue des responsables du S.V.T. qui avaient initialement misé sur des voitures à impériales construites par la firme Béchade à Ivry sur le modèle de celles des Tramways de Paris, à traction animale ! Il a fallu les reconstruire dès avant l'inauguration de la ligne... 